\title{
LETTER
}

\section{Plasma tau levels in Alzheimer's disease}

\author{
Henrik Zetterberg*1,2, David Wilson³, Ulf Andreasson', Lennart Minthon ${ }^{4}$, Kaj Blennow', Jeffrey Randall ${ }^{3}$ \\ and Oskar Hansson ${ }^{4}$
}

\section{Introduction}

Efforts to find reliable blood biomarkers for Alzheimer's disease $(\mathrm{AD})$ in a highly warranted clinical laboratory test have met with little success. There is no clear change in plasma $\beta$-amyloid in $A D$, and assays for the axonal injury marker tau have been hampered by a lack of analytical sensitivity for accurate measurement in blood samples [1]. Here, the results of a novel ultra-sensitive assay for tau in peripheral blood are reported.

\section{Findings}

We have developed an ultra-sensitive assay for tau in peripheral blood [2]. In brief, the assay is based on digital array technology [3] and uses the Tau5 monoclonal antibody for capture (Covance, Princeton, NJ, USA) and HT7 and BT2 monoclonal antibodies for detection (Pierce, now part of Thermo Fisher Scientific Inc., Waltham, MA, USA). This combination reacts with both normal and phosphorylated tau with epitopes in the mid-region of the molecule, making the assay sensitive to all known tau isoforms. The calibrator was recombinant tau 381 (EMD Millipore Corporation, Billerica, MA, USA). To minimize matrix effects, all samples were diluted 1:4 in phosphatebuffered saline with $2 \%$ bovine serum albumin diluent prior to assay. The limit of detection of the assay, which requires $30 \mu \mathrm{L}$ of plasma, is $0.02 \mathrm{pg} / \mathrm{mL}$ [2], which is more than 1,000 -fold more sensitive than conventional immunoassays.

Here, we assess the association of plasma tau levels with $\mathrm{AD}$ in a cross-sectional study of 54 patients with $\mathrm{AD}$ dementia [4], 75 patients with mild cognitive impairment (MCI) [5], and 25 cognitively normal controls (Table 1). All participants were recruited at the specialized memory clinic at Skåne University Hospital in Malmö, Sweden, and underwent extensive clinical evaluation, including cerebrospinal fluid (CSF) sampling by lumbar puncture,

\footnotetext{
*Correspondence: henrik.zetterberg@gu.se

${ }^{1}$ Clinical Neurochemistry Laboratory, Institute of Neuroscience and Physiology, Department of Psychiatry and Neurochemistry, the Sahlgrenska Academy at the University of Gothenburg, S-431 80 Mölndal, Sweden

Full list of author information is available at the end of the article
}

Table 1. Demographic and biochemical data

\begin{tabular}{lccc}
\hline & $\begin{array}{c}\text { AD } \\
(\mathbf{n = 5 4 )}\end{array}$ & $\begin{array}{c}\text { MCI } \\
(\mathbf{n = 7 5 )}\end{array}$ & $\begin{array}{c}\text { Controls } \\
(\mathbf{n}=\mathbf{2 5})\end{array}$ \\
\hline Age, years & $75(6.2)$ & $68(9.3)$ & $74(6.7)$ \\
Gender, male/female & $17 / 37$ & $29 / 46$ & $6 / 19$ \\
MMSE, score & $19(4.9)^{\mathrm{a}}$ & $27(1.6)$ & $29(1.4)$ \\
Plasma T-tau, pg/mL & $8.80(10.1)^{\mathrm{b}, c}$ & $4.68(4.25)$ & $4.43(2.83)$ \\
CSFT-tau, pg/mL & $828(375)^{\mathrm{a}}$ & $550(421)$ & $507(254)$ \\
CSF P-tau, pg/mL & $123(49.2)^{\mathrm{a}}$ & $78.1(28.8)$ & $73.4(20.5)$ \\
CSF A $42, \mathrm{pg} / \mathrm{mL}$ & $380(87.6)^{\mathrm{a}}$ & $478(204)$ & $584(215)$ \\
\hline
\end{tabular}

Quantitative data are presented as mean (standard deviation). Statistical differences were determined by using nonparametric tests. Cerebrospinal fluid (CSF) biomarker concentrations are INNOTEST ELISA-normalized Luminex AlzBio3 (Innogenetics, Gent, Belgium) values. ${ }^{a}$ Compared with patients with mild cognitive impairment $(\mathrm{MCl})$ or controls, $P<0.001$. ${ }^{\circ}$ Compared with patients with $\mathrm{MCl}, P=0.001$. 'Compared with controls, $P=0.02$. AD, Alzheimer's disease; MMSE, mini-mental state examination.

in addition to venipuncture and collection of blood in ethylenediaminetetraacetic acid (EDTA) tubes for plasma preparation by centrifugation within 15 minutes from sampling. Plasma samples were aliquoted into cryo tubes and stored at $-80^{\circ} \mathrm{C}$ pending analysis, which was performed on one occasion by using one batch of reagents with an average coefficient of variation of 9.7\% for triplicate measurements of each sample. The patients with MCI were cognitively stable for an average of 101 months $(n=36)$ or developed AD dementia $(n=35)$ or other types of dementias - vascular dementia $(n=3)$ and semantic dementia $(n=1)$ - during follow-up. The study was approved by the regional ethics committee at Lund University and complied with the Declaration of Helsinki. Informed consent was obtained from all study participants.

Tau levels in plasma were significantly higher in AD patients compared with both controls and $\mathrm{MCI}$ patients (Figure 1a). MCI patients who developed AD during follow-up had tau levels similar to those of patients with stable MCI and cognitively normal controls (Figure $1 \mathrm{~b}$ ). There was no correlation between tau levels in plasma and CSF in any diagnostic group (Figure 1c).

The results of this study have several important implications. First, plasma tau levels are elevated in AD but with overlapping ranges across diagnostic groups. 


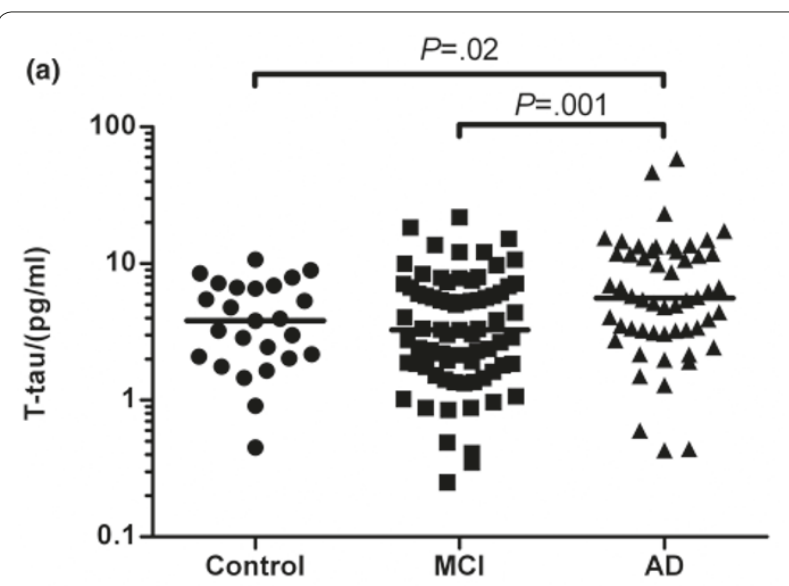

(b)

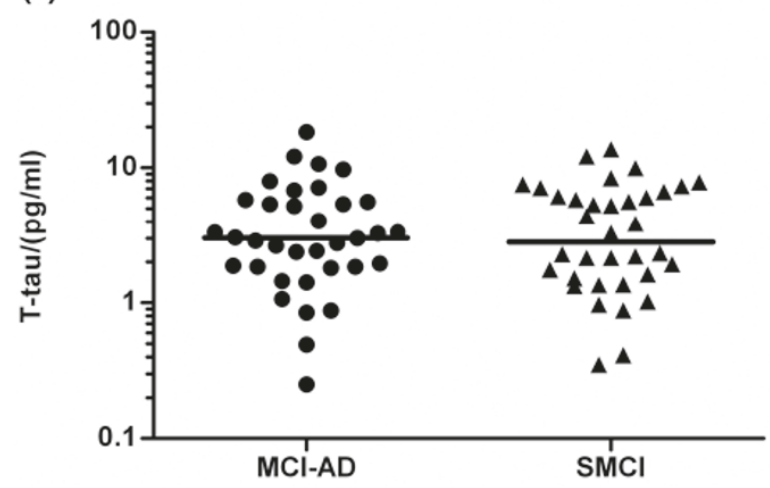

(c)



Figure 1. Elevated tau levels in plasma from patients with Alzheimer's disease (AD). (a) Plasma levels of tau are elevated in patients with $\mathrm{AD}$ compared with cognitively normal controls and patients with mild cognitive impairment (MCl). (b) MCl patients who developed $\mathrm{AD}$ (MCI-AD) during follow-up had baseline tau levels similar to those of patients with stable $\mathrm{MCl}(\mathrm{SMCl})$. (c) There was no correlation between tau levels in plasma and cerebrospinal fluid (CSF) in any diagnostic group. Thin horizontal lines in panels (a) and (b) indicate medians. A nonparametric Kruskal-Wallis test followed by Mann-Whitney was performed to test for statistical significance. Spearman's rank correlation coefficient was used to assess the relationship between plasma and CSF tau concentrations in panel (c), where open circles, gray squares, and black triangles represent AD, $\mathrm{MCl}$, and controls, respectively.
This overlap diminishes the utility of plasma tau as a diagnostic test. However, further studies are needed to evaluate plasma tau as a first-in-line screening tool (for example, in the primary care setting and perhaps together with other markers in a biomarker panel). Second, normal plasma tau levels in the MCI stage of AD suggest that plasma tau is a late marker, requiring substantial axonal injury before increasing to abnormal levels. In this context, other neurodegenerative diseases (for example, Creutzfeldt-Jakob disease) as well as acute conditions (for example, stroke and brain trauma) should be tested. Third, the lack of correlation of tau levels in plasma and CSF suggests that steady-state concentrations of tau in these two body fluids are differentially regulated. In our earlier study of patients with hypoxic brain injury following cardiac arrest, tau was rapidly (within 24 hours) cleared from blood in patients with good neurological outcome [2], indicating potent clearance mechanisms for this marker in the bloodstream. This may obscure any correlation with CSF tau levels, which stay elevated for weeks following an acute neurological insult [6].

This article is part of a series on Peripheral Biomarkers, edited by Douglas Galasko. Other articles in this series can be found at http://alzres.com/series/biomarkers

\section{Abbreviations}

AD, Alzheimer's disease; CSF, cerebrospinal fluid; MCl, mild cognitive impairment.

\section{Competing interests}

DW and JR, who are employees of Quanterix Corporation (Lexington, MA, USA), and $\mathrm{HZ}$ and $\mathrm{KB}$ are listed as inventors on a US patent application for plasma tau as a brain injury marker. The other authors declare that they have no competing interests.

\section{Acknowledgments}

This study was funded by grants from Swedish Brain Power, the Swedish Research Council, the Wolfson Foundation, the Alzheimer's Association, the JPND Project BIOMARKAPD, Swedish State Support for Clinical Research, the Swedish Brain Fund, the Alzheimer Foundation of Sweden, and the Dementia Association of Sweden.

\section{Author details}

'Clinical Neurochemistry Laboratory, Institute of Neuroscience and Physiology, Department of Psychiatry and Neurochemistry, the Sahlgrenska Academy at the University of Gothenburg, S-431 80 Mölndal, Sweden. ${ }^{2} U C L$ Institute of Neurology, Queen Square, London, WC1N 3BG, UK. ${ }^{3}$ Quanterix Corporation, 113 Hartwell Avenue, Lexington, MA 02421, USA. ${ }^{4}$ Clinical Memory Research Unit, Clinical Sciences Malmö, Lund University, Simrisbanvägen 14, S-212 24 Malmö, Sweden.

Published: 28 March 2013

\section{References}

1. Blennow K, Hampel H, Weiner M, Zetterberg H: Cerebrospinal fluid and plasma biomarkers in Alzheimer disease. Nat Rev Neurol 2010, 6:131-144.

2. Randall J, Mörtberg E, Provuncher GK, Fournier DR, Duffy DC, Rubertsson S, Blennow K, Zetterberg $\mathrm{H}$, Wilson DH: Tau proteins in serum predict neurological outcome after hypoxic brain injury from cardiac arrest: results of a pilot study. Resuscitation 2012 Aug 9. [Epub ahead of print].

3. Rissin DM, Kan CW, Campbell TG, Howes SC, Fournier DR, Song L, Piech T, Patel PP, Chang L, Rivnak AJ, Ferrell EP, Randall JD, Provuncher GK, Walt DR, 
Duffy DC: Single-molecule enzyme-linked immunosorbent assay detects serum proteins at subfemtomolar concentrations. Nat Biotechnol 2010,

28:595-599.

4. McKhann G, Drachman D, Folstein M, Katzman R, Price D, Stadlan EM: Clinical diagnosis of Alzheimer's disease: report of the NINCDS-ADRDA Work Group under the auspices of Department of Health and Human Services Task Force on Alzheimer's Disease. Neurology 1984, 34:939-944.

5. Winblad B, Palmer K, Kivipelto M, Jelic V, Fratiglioni L, Wahlund LO, Nordberg A, Bäckman L, Albert M, Almkvist O, Arai H, Basun H, Blennow K, de Leon M, DeCarli C, Erkinjuntti T, Giacobini E, Graff C, Hardy J, Jack C, Jorm A, Ritchie K, van Duijn C, Visser P, Petersen RC: Mild cognitive impairment - beyond controversies, towards a consensus: report of the International Working Group on Mild Cognitive Impairment. J Intern Med 2004, 256:240-246.

6. Hesse C, Rosengren L, Andreasen N, Davidsson P, Vanderstichele H, Vanmechelen E, Blennow K: Transient increase in total tau but not phospho-tau in human cerebrospinal fluid after acute stroke. Neurosci Lett 2001, 297:187-190.

doi:10.1186/alzrt163

Cite this article as: Zetterberg $\mathrm{H}$, et al:: Plasma tau levels in Alzheimer's

disease. Alzheimer's Research \& Therapy 2013, 5:9. 